\title{
ON TWO FUNCTION SPACES WHICH ARE SIMILAR TO $L_{0}$
}

\author{
S. J. DILWORTH AND D. A. TRAUTMAN
}

(Communicated by William J. Davis)

ABSTRACT. Let $\Lambda_{0}$ consist of all functions $f$ measurable on $(0, \infty)$ with

$$
\lambda\{s:|f(s)|>t\}<\infty
$$

for all $t>0$, where $\lambda$ is Lebesgue measure, and let $L_{0}(0, \infty)$ consist of all measurable functions $f$ with

$$
\lim _{t \rightarrow \infty} \lambda\{s:|f(s)|>t\}=0 .
$$

Let each space have the topology induced by convergence in measure. We show that every infinite-dimensional Banach subspace of $\Lambda_{0}$ contains $c_{0}$ or $l_{p}$ for some $1 \leq p<\infty$. We also identify the duals of $\Lambda_{0}$ and $L_{0}(0, \infty)$.

\section{INTRODUCTION AND NOTATION}

Let $\mathscr{L}_{0}(0, \infty)$ denote the collection of all (equivalence classes of) almost everywhere finite measurable functions on $(0, \infty)$, and let $L_{0}(0, \infty)$ denote the collection of all $f \in \mathscr{L}_{0}(0, \infty)$ satisfying $\lambda(\{s:|f(s)|>t\}) \rightarrow 0$ as $t \rightarrow \infty$, where $\lambda$ is Lebesgue measure. $L_{0}(0, \infty)$ is a topological vector space under the topology of convergence in measure, and it is the largest linear subspace of $\mathscr{L}_{0}(0, \infty)$ with this property. Routine calculations show that $L_{0}(0, \infty)$ is a non-separable complete metric linear space, with the metric given by $d(f, g)=$ $\|f-g\|$, where

$$
\|f\|=\sup _{E} \int_{E} \frac{|f|}{1+|f|} d \lambda,
$$

the supremum being taken over all $E \subset(0, \infty)$ of measure one.

The distribution function $d_{f}(t)$ of a measurable function $f$ on $(0, \infty)$ is defined by

$$
d_{f}(t)=\lambda(\{s:|f(s)|>t\}) \quad(0<t<\infty),
$$

and the decreasing rearrangement $f^{*}(t)$ by

$$
f^{*}(t)=\inf \left\{s>0: d_{f}(s) \leq t\right\} .
$$

Received by the editors April 10, 1989.

1980 Mathematics Subject Classification (1985 Revision). Primary 46A06, 46E30; Secondary 46B15, 46A45.

The first author was supported in part by NSF DMS8801731. 
We shall study the separable subspace of $L_{0}(0, \infty)$ consisting of all $f \in$ $L_{0}(0, \infty)$ for which $d_{f}(t)<\infty$ for all $t>0$. In view of the classical identity $\|f\|_{\Lambda_{\alpha}}=\left\|d_{f}\right\|_{L_{\alpha}}^{\alpha}(0<\alpha<\infty)$, where $L_{\alpha}$ and $\Lambda_{\alpha}$ are the standard Lebesgue and Lorentz function spaces (see [10] for the original definition of $\Lambda_{\alpha}$ ), it seems to be appropriate to denote the collection of all functions on $(0, \infty)$ with a finite distribution function by the symbol $\Lambda_{0}$, and we have chosen to do so.

In $\S 2$ the Banach subspaces of $\Lambda_{0}$ are investigated. It is proved that $\Lambda_{0}$ contains an isomorphic copy of every Orlicz function space on $(0, \infty)$. The main result of the paper is Theorem 2.3, which says that every infinite-dimensional Banach subspace of $\Lambda_{0}$ contains a subspace isomorphic to $c_{0}$ or to $l_{p}(1 \leq p<$ $\infty)$. The prototype for results of this kind, of course, is the famous theorem of D. J. Aldous [1] that every infinite-dimensional subspace of $L_{1}(0,1)$ contains $l_{p}$ for some $1 \leq p \leq 2$. Subsequently, various generalizations of Aldous's theorem have been obtained (see e.g. [5]). Our strategy in proving Theorem 2.3 is perhaps the obvious one of reducing the problem to the consideration of Banach spaces whose subspace structure is already clearly understood.

In the short third section the dual spaces of $\Lambda_{0}$ and $L_{0}(0, \infty)$ are computed. It is proved that $\Lambda_{0}$ has trivial dual and that the dual of $L_{0}(0, \infty)$ may be identified with an infinite-dimensional subspace of the dual of $L_{\infty}(0, \infty)$.

Let us now state some definitions and notation which we shall use in the remainder of the paper. An Orlicz function $\phi:[0, \infty) \rightarrow[0, \infty)$ is a strictly increasing function, continuous at 0 , with $\phi(0)=0$. We say that $\phi$ satisfies the $\Delta_{2}$-condition at 0 if $\sup _{0<x \leq 1} \phi(2 x) / \phi(x)<\infty$. We define the Orlicz function space $L_{\phi}(0, \infty)$ as the space of all functions $f$ measurable on $(0, \infty)$ with

$$
\int_{0}^{\infty} \phi\left(\frac{|f(t)|}{\alpha}\right) d t<\infty
$$

for all $\alpha>0$. The topology is given by the quasi-norm

$$
\|f\|_{\phi}=\inf \left\{\alpha>0: \int_{0}^{\infty} \phi\left(\frac{|f(t)|}{\alpha}\right) d t \leq 1\right\} .
$$

The Orlicz sequence space $l_{\phi}$ is defined as the space of all sequences $\left(a_{n}\right)$ such that

$$
\sum_{n=1}^{\infty} \phi\left(\frac{\left|a_{n}\right|}{\alpha}\right)<\infty
$$

for all $\alpha<\infty$. We use a quasi-norm similar to that of $L_{\phi}(0, \infty)$ to define the topology.

In a topological vector space $X$, a sequence $\left(x_{n}\right)$ is a basis for $X$ if, for each $x \in X$, there exist unique scalars $\left(a_{n}\right)$ with

$$
x=\sum_{n=1}^{\infty} a_{n} x_{n} .
$$


A basic sequence $\left(x_{n}\right)$ is a sequence which is a basis for its closed linear span $\overline{\operatorname{lin}}\left(x_{n}\right)$. Two bases $\left(x_{n}\right)$ of $X$ and $\left(y_{n}\right)$ of $Y$ are equivalent whenever $\sum_{n=1}^{\infty} a_{n} x_{n}$ converges if and only if $\sum_{n=1}^{\infty} a_{n} y_{n}$ converges. A basis $\left(x_{n}\right)$ of $X$ is symmetric if for each permutation $\pi$ of $\mathbf{Z}^{+},\left(x_{\pi(n)}\right)$ is equivalent to $\left(x_{n}\right)$.

If $K$ is a subset of a topological vector space, $\operatorname{conv}(K)$ will denote its convex hull. In $\Lambda_{0}$ or $L_{0}(0, \infty)$, for $\varepsilon>0$ we let

$$
B_{\varepsilon}=\{f:\|f\|<\varepsilon\} .
$$

The indicator function of a set $E$ is denoted by $I(E)$. The reader should consult [8] and [9] for other standard notation and terminology.

\section{SubSPACES OF $\Lambda_{0}$}

Our first proposition shows that $\Lambda_{0}$ has a very large collection of Banach and quasi-Banach subspaces. This observation lends interest to the main result, Theorem 2.3 below, and provides a motivation for wishing to prove it.

Proposition 2.1. Let $\phi$ be an Orlicz function. Then $L_{\phi}(0, \infty)$ is isomorphic to a subspace of $\Lambda_{0}$.

Proof. Since $\Lambda_{0}$ is clearly isomorphic to the space $\tilde{\Lambda}_{0}$ consisting of all measurable functions on $(0, \infty) \times(0, \infty)$ possessing a distribution function, it will be enough to show that $\tilde{\Lambda}_{0}$ contains a subspace isomorphic to $L_{\phi}(0, \infty)$. To this end, let $f(s)=\left(\phi^{-1}(s)\right)^{-1}$, and define $T: L_{\phi}(0, \infty) \rightarrow \tilde{\Lambda}_{0}$ by $g(t) \mapsto f(s) g(t)$. To see that $T$ maps $L_{\phi}(0, \infty)$ into $\tilde{\Lambda}_{0}$, observe that $g \in L_{\phi}(0, \infty)$ if and only if, for all $\alpha>0$, we have

$$
\int_{0}^{\infty} \phi\left(\frac{|g(t)|}{\alpha}\right) d t<\infty
$$

that is, if and only if

$$
\int_{0}^{\infty} f^{-1}\left(\frac{\alpha}{|g(t)|}\right) d t<\infty
$$

This is the case if and only if

$$
\int_{0}^{\infty} \lambda(\{s: f(s)|g(t)|>\alpha\}) d t<\infty
$$

for all $\alpha>0$. By Fubini's theorem, the latter condition is simply the statement that $f(s) g(t)$ belongs to $\tilde{\Lambda}_{0}$. It is also easy to check from the above calculation that $T$ is an isomorphism onto its range.

The main calculations used in the proof of Theorem 2.3 are brought together in the following lemma. There is a close affinity between this lemma and [ 3 , Proposition 2.3]. 
Lemma 2.2. Let $X$ be a Banach space and let $T: X \rightarrow \Lambda_{0}$ be an isomorphic embedding. For each $M>0$, let $\psi_{M}(f)=f \cdot I(0, M)$. If, for each $M>0$, $\psi_{M} \circ T$ does not restrict to an isomorphic embedding of any infinite-dimensional subspace of $X$, then $X$ contains a symmetric basic sequence.

Proof. By passing to a subspace, if necessary, we may assume that $X$ has a normalized Schauder basis $\left(x_{n}\right)_{n=1}^{\infty}$. Let $\varepsilon>0$ be given. Since $\psi_{M} \circ T$ is not an isomorphism on any infinite-dimensional subspace of $X$ for any $M$, one can find by induction a normalized block basic sequence $\left(y_{n}\right)_{n=1}^{\infty}$ of $\left(x_{n}\right)_{n=1}^{\infty}$ and an increasing sequence $\left(M_{n}\right)_{n=1}^{\infty}$ of positive numbers such that $\left\|T\left(y_{n}\right) \cdot I\left(0, M_{n-1}\right)\right\|<\varepsilon / 2^{n}$ and $\left\|T\left(y_{n}\right) \cdot I\left(M_{n}, \infty\right)\right\|<\varepsilon / 2^{n}$. Let $z_{n}=$ $T\left(y_{n}\right) \cdot I\left(M_{n-1}, M_{n}\right)$. Provided $\varepsilon>0$ is chosen so small that $\inf \left\{\left\|z_{n}\right\|: 1 \leq\right.$ $n<\infty\}>0$, [6, Lemma 4.3] shows that $\left(z_{n}\right)_{n=1}^{\infty}$ is a basic sequence in $\Lambda_{0}$ equivalent to $\left(T\left(y_{n}\right)\right)_{n=1}^{\infty}$. Select measurable subsets $A_{n} \subset\left(M_{n-1}, \infty\right)$ such that $\lambda\left(A_{n}\right)=1$ and $\operatorname{ess} \inf \left\{\left|z_{n}(t)\right|: t \in A_{n}\right\} \geq \operatorname{ess} \sup \left\{\left|z_{n}(t)\right|: t \in A_{n}^{c}\right\}$, and let $z_{n}^{\prime}=z_{n} \cdot I\left(A_{n}\right)$. It is easily seen that $\left\|\sum_{n=1}^{\infty} a_{n} z_{n}\right\|=\left\|\sum_{n=1}^{\infty} a_{n} z_{n}^{\prime}\right\|$ for all scalars $\left(a_{n}\right)_{n=1}^{\infty}$, and so $\left(z_{n}\right)_{n=1}^{\infty}$ and $\left(z_{n}^{\prime}\right)_{n=1}^{\infty}$ are equivalent basic sequences. Let $\delta=\inf \left\{\left\|z_{n}^{\prime}\right\|: 1 \leq n<\infty\right\}$ and let $Z_{n}$ be the decreasing rearrangement of $z_{n}^{\prime}$. By passing to a subsequence and relabeling, we may assume by Helly's selection theorem that $Z_{n} \rightarrow Z$ pointwise, where $Z$ is a decreasing function supported on $[0,1]$. By Egorov's theorem we may also assume, after passing to a further subsequence, that $\left|Z_{n}-Z\right|<\delta / 4^{n}$ except on a set of measure less than $\delta / 4^{n}$. By [2, Theorem 7.5], for each $n$ there exists a measure-preserving transformation $\sigma_{n}: A_{n} \rightarrow[0,1]$ with $\left|z_{n}^{\prime}\right|=Z_{n} \circ \sigma_{n}$ almost everywhere. Let $W_{n}=\left(Z \circ \sigma_{n}\right) \cdot \operatorname{sgn}\left(z_{n}^{\prime}\right)$. Then $\left\|z_{n}^{\prime}-W_{n}\right\|<2 \delta / 4^{n}$, and so by [6, Lemma 4.3] $\left(W_{n}\right)_{n=1}^{\infty}$ and $\left(z_{n}^{\prime}\right)_{n=1}^{\infty}$ are equivalent basic sequences. Clearly $\left(W_{n}\right)_{n=1}$ is a symmetric basic sequence as each $W_{n}$ has $Z$ as its decreasing rearrangement. Remark. It is easy to see that if the function $Z$ of the previous proof is bounded, then $\left(W_{n}\right)_{n=1}^{\infty}$ spans a subspace isomorphic to $c_{0}$. Since $c_{0}$ does not embed isomorphically into $L_{0}(0,1)$ it follows that $\Lambda_{0}$ and $L_{0}(0,1)$ are not isomorphic spaces. (The latter fact also follows from Proposition 2.1.) In particular, $\Lambda_{0}$ has at least two non-isomorphic complemented subspaces.

Theorem 2.3. Let $X$ be a Banach subspace of $\Lambda_{0}$. Then $X$ contains a subspace isomorphic to $c_{0}$ or to $l_{p}$ for some $1 \leq p<\infty$.

Proof. Suppose that $T: X \rightarrow \Lambda_{0}$ is an isomorphic embedding. If, for some $M$, $\phi_{M} \circ T$ restricts to an isomorphic embedding, then some infinite-dimensional subspace of $X$ is by dilation isomorphic to a subspace of $L_{0}(0,1)$, and thus isomorphic to a subspace of $L_{q}(0,1)$ for all $0<q<1$ (see [11]). One can then modify Garling's work ([5, Theorem 16, p. 167, Theorem 7, p. 138 and Theorem 6, p. 136]) to show that $L_{q}(0,1)$ is stable, that all stable $q$-Banach spaces have an $l_{p}$-type for some $q \leq p<\infty$, and that all infinite-dimensional stable $q$-Banach spaces contain an isomorphic copy of $l_{p}$ for some $q \leq p<\infty$. 
Since $X$ is a Banach space it follows that $X$ must contain a copy of $l_{p}$ for some $1 \leq p<\infty$.

Now suppose that $X$ is a Banach subspace of $\Lambda_{0}$ satisfying the hypotheses of Lemma 2.2. Then $X$ contains a subspace isomorphic to the closed linear span of a sequence of functions $\left(f_{n}\right)_{n=1}^{\infty}$, each having a common distribution function $d_{f}(t)$. By the remark following Lemma 2.2 we may assume that $f$ is an unbounded function. Let $\phi(t)=d_{f}(1 / t)$. A short calculation shows that $\sum_{n=1}^{\infty} a_{n} f_{n}$ converges in $\Lambda_{0}$ if and only if $\sum_{n=1}^{\infty} \phi\left(\frac{\left|a_{n}\right|}{\lambda}\right)<\infty$ for all $\lambda>0$. Hence $\overline{\operatorname{lin}}\left(f_{n}\right)$ and $l_{\phi}$ are isomorphic spaces. By aping the proof of [9, Proposition 4.a.4], we see that either $l_{\phi}$ contains $c_{0}$ or $\phi$ satisfies the $\Delta_{2}$ condition at 0 . If $\phi$ satisfies the $\Delta_{2}$-condition at 0 , then by [7, Theorem 3.3] $l_{\phi}$ is isomorphic to $l_{\psi}$ for some convex Orlicz function $\psi$ which satisfies the $\Delta_{2}$ condition at 0 . An appeal to [9, Theorem 4.a.9] shows that $l_{\psi}$, and hence both $l_{\phi}$ and $\overline{\operatorname{lin}}\left(f_{n}\right)$, contains an isomorphic copy of $c_{0}$ or $l_{p}$ for some $1 \leq p<\infty$.

\section{The dual of $L_{0}(0, \infty)$}

We begin by showing that $\Lambda_{0}$ has trivial dual. The proof is modelled on Day's proof [4] that $L_{p}$ has trivial dual for $0<p<1$.

Proposition 3.1. $\Lambda_{0}^{*}=\{0\}$.

Proof. Let $\varepsilon>0$; we shall show that $\Lambda_{0}=\operatorname{conv} B_{\varepsilon}$. Let $f \in \Lambda_{0}$ and let $A=\{t:|f(t)| \geq \varepsilon / 2\}$. Since $\lambda(A)<\infty$ we may decompose it into finitely many sets $A_{i}(1 \leq i \leq n)$ of measure less than $\varepsilon / 2$. Let $f_{i}=n f \cdot I\left(A_{i}\right)+f \cdot I\left(A^{c}\right)$. Then each $f_{i} \in B_{\varepsilon}$ and $f=\sum_{i=1}^{n}(1 / n) f_{i}$. It follows that $\Lambda_{0}$ has trivial dual.

Two easy lemmas are required to identify the dual of $L_{0}(0, \infty)$.

Lemma 3.2. Let $\mathcal{N}$ be a neighborhood base of the origin for $L_{0}(0, \infty)$. Then $\Lambda_{0}=\cap_{U \in \mathscr{N}} \operatorname{conv} U$.

Proof. The fact that $\Lambda_{0} \subset \cap_{U \in \mathcal{N}}$ conv $U$ was essentially proved in Proposition 3.1. Let $f \in L_{0}(0, \infty) \backslash \Lambda_{0}$; then there exists $a>0$ such that $\lambda\{t:|f(t)|>a\}=$ $\infty$. Let $0<\varepsilon<a /(1+a)$, let $f_{1}, \ldots, f_{n} \in B_{\varepsilon}$, and let $E_{i}=\left\{t:\left|f_{i}(t)\right| \geq a\right\}$. Clearly $\lambda\left(E_{i}\right) \leq 1$, and so $\lambda\left\{t:\left|\left(\sum_{i=1}^{n} \alpha_{i} f_{i}\right)(t)\right| \geq a\right\} \leq n$ whenever $\alpha_{i} \geq 0$, $\sum_{i=1}^{n} \alpha_{i}=1$. Thus $f \neq \sum_{i=1}^{n} a_{i} f_{i}$ for any such $\alpha_{i}$, and so $f \notin \operatorname{conv}\left(B_{\varepsilon}\right)$. It follows that $\cap_{U \in \mathfrak{A}}$ conv $U \subset \Lambda_{0}$.

Lemma 3.3. The space $L_{0}(0, \infty) / \Lambda_{0}$ is isomorphic to the Banach space $L_{\infty}(0, \infty) /\left(\Lambda_{0} \cap L_{\infty}(0, \infty)\right)$.

Proof. Let $f \in L_{0}(0, \infty)$. Then there exists $M>0$ such that $\lambda\{t:|f(t)|>$ $M\}<\infty$. Clearly $f \cdot I\{t:|f(t)| \leq M\} \in L_{\infty}(0, \infty)$, while $f \cdot I\{t:|f(t)|>M\} \in$ $\Lambda_{0}$, and so the mapping $T: L_{\infty}(0, \infty) \rightarrow L_{0}(0, \infty) / \Lambda_{0}$ given by $T(g)=g+\Lambda_{0}$ 
is onto. $T$ is clearly continuous and its kernel is $\Lambda_{0} \cap L_{\infty}(0, \infty)$, whence the result.

Theorem 3.4. The dual of the space $L_{0}(0, \infty)$ may be identified with the dual of the Banach space $L_{\infty}(0, \infty) /\left(\Lambda_{0} \cap L_{\infty}(0, \infty)\right)$.

Proof. By Lemma 3.2 every element of $\left(L_{0}(0, \infty)\right)^{*}$ vanishes on $\Lambda_{0}$. The result now follows from Lemma 3.3.

Remarks. 1. Theorem 3.4 allows an explicit description of the dual of $L_{0}(0, \infty)$. Let $\psi \in\left(L_{0}(0, \infty)\right)^{*}$. Then there exists a finitely additive signed measure $\mu_{\psi}$ on the Lebesgue $\sigma$-field of $(0, \infty)$ such that $\mu_{\psi}(A)=0$ for every $A \subset(0, \infty)$ with $\lambda(A)<\infty$, and such that for all $f \in L_{0}(0, \infty)$ we have

$$
\psi(f)=\lim _{M \rightarrow \infty} \int(f \wedge M) d \mu_{\psi}
$$

where $(f \wedge M)(t)=\min (f(t), M)$. Conversely, every such measure $\mu$ defines a continuous linear functional on $L_{0}(0, \infty)$.

2. It is possible to show that there is no continuous linear projection from $L_{0}(0, \infty)$ onto $\Lambda_{0}$ by adapting Whitley's proof [12] of the non-existence of a bounded projection from $l_{\infty}$ onto $c_{0}$. We omit the details of this result.

\section{REFERENCES}

1. D. J. Aldous, Subspaces of $L^{1}$ via random measures, Trans. Amer. Math. Soc. 267 (1981), 445-463.

2. C. Bennett and R. C. Sharpley, Interpolation of operators, Academic Press, New York, 1988.

3. N. L. Carothers and S. J. Dilworth, Subspaces of $L_{p, q}$, Proc. Amer. Math. Soc. 104 (1988), 537-545.

4. M. M. Day, The spaces $L_{p}$ with $0<p<1$, Bull. Amer. Math. Soc. 46 (1940), 816-823.

5. D. J. H. Garling, Stable Banach spaces, random measures and Orlicz function spaces, in Probability Measures on Groups (Proceedings Oberwolfach), Lecture Notes 928, A. Dold and B. Eckmann, eds., Springer-Verlag, New York, 1981, 121-175.

6. N. J. Kalton, Basic sequences in F-spaces and their applications, Proc. Edinburgh Math. Soc. 19 (1974), 151-167.

7. $ـ$, Orlicz sequence spaces without local convexity, Math. Proc. Camb. Phil. Soc. 81 (1977), 253-277.

8. N. J. Kalton, N. T. Peck and James W. Roberts, An F-space sampler, London Math. Soc. Lecture Notes Series 89, Cambridge University Press, Cambridge, England, 1984.

9. J. Lindenstrauss and L. Tzafriri, Classical Banach spaces I, Sequence spaces, Springer-Verlag, 1977.

10. G. G. Lorentz, Some new functional spaces, Ann. Math. 51 (1950), 37-55.

11. E. M. Nikishin, Resonance theorems and superlinear operators, Usp. Matem. Nauk. 25 (1970), 129-191 (= Russian Math. Surveys 25 (1970), 124-187).

12. R. J. Whitley, Projecting $m$ onto $c_{0}$, Amer. Math. Monthly 73 (1966), 285-286.

University of South Carolina, Department of Mathematics, Columbia, South CarOLINA 29208

The Citadel, Department of Mathematics and Computer Science, Charleston, South Carolina 29409 